Results 1656 subjects were seen (Active TB: 22\%, Latent TB: $11 \%$, Other final diagnosis not TB: $67 \%$ ). 748 (45\%) had all three $\mathrm{BBV}$ tests performed. This was significantly different between the groups, as subjects with either latent or active TB were more likely to have all three tests (Active: $90 \%$, Latent: $83 \%$ vs Other diagnosis: $24 \%, \mathrm{P}<0.001$ ). In those tested, an HIV positive result was more frequently found in active TB (7.5\%), whilst the HIV prevalence was similar in the two other groups (Latent $1.3 \%$ vs Other $1.4 \%$ ). HBV was more common in Active \& Latent TB populations compared to the Others ( $3 \%$ vs $0.8 \%, \mathrm{P}<0.001)$. HCV had a similar prevalence pattern to HIV, in that it was more common in those with Active TB (3\% vs Latent TB: $0.6 \%$, Other $1 \%$, $\mathrm{P}=0.06)$.

Conclusion We find a high rate of uptake for HIV, HBV \& HCV testing in our subjects treated for active and latent TB. HIV \& HCV were more common in the former population. Adults with latent TB had a similar prevalence of HIV and HCV to those subjects whose final diagnosis was other than TB. However, the latter had a much lower overall uptake of BBV testing. This is of concern and suggests that we are missing opportunities to diagnose unsuspected $\mathrm{BBV}$ infection in a large number of clinic attendees.

\section{P55 IDENTIFYING POTENTIAL PREDICTORS OF MORTALITY AND MORBIDITY IN TUBERCULOUS MENINGITIS (TBM)}

doi:10.1136/thoraxjnl-2012-202678.196

C Petridou, C Petridou, A Jayaratnam. Barking, Havering Redbridge, NHS Trust, Ilford, United Kingdom

Introduction The early and prompt diagnosis of TBM is essential to improve outcomes. Delayed treatment leads to increased morbidity and mortality. Cerebrospinal fluid (CSF) findings may assist in early diagnosis as well as potentially identifying patients at highrisk of death

Methods A retrospective analysis of patients treated for TBM from 2000-2010 was undertaken using the London TB Register. Data was collected from medical records and pathology results.

Results Mortality was 20\% (8/41) and 12\% (5/41) were left with permanent neurological deficits. Mycobacterium tuberculosis (MTB) was cultured from CSF in 37\% (14/38). Mortality in patients in whom CSF was culture positive was higher than those whose CSF culture was negative ( $33 \%$ vs $16 \%$ ). The mean value of CSF protein was higher in patients with poorer outcomes than those who recovered fully $(2.72 \mathrm{vs} 1.92 \mathrm{~g} / \mathrm{L})$.

Corticosteroids were given to $92 \%$ of patients (35/38). Of the three patients who did not receive steroids, one was left with a permanent disability.

Conclusions In our population, we observed high mortality and morbidity rates for patients diagnosed with TBM. A large proportion of diagnoses were not confirmed microbiologically. Higher mortality rates were observed in those whose CSF cultured MTB A possible explanation for this finding is that those patients for whom microbiological confirmation was not obtained, the presumptive diagnosis of TBM was not correct. There is a trend towards higher mortality and morbidity in those with higher CSF protein values. This may be an indicator of increased bacterial load with an associated increase in inflammatory cytokines.

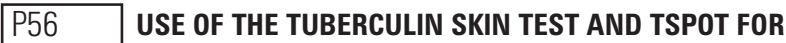 SCREENING PRIOR TO TNF ANTAGONIST THERAPY IDENTIFIES ADDITIONAL PATIENTS ELIGIBLE FOR CHEMOPROPHYLAXIS COMPARED TO USE OF RISK ASSESSMENT STRATEGIES ALONE}

doi:10.1136/thoraxjnl-2012-202678.197

K Manalan, A Singanayagam, PL Molyneaux, PM George, DW Connell, A Lalvani, M Wickremasinghe, OM Kon. St. Mary's Hospital, London, UK
Patients with rheumatological disease who are treated with TNF antagonist therapy are at increased risk of reactivating latent tuberculosis infection (LTBI). It is believed that immunosuppressant therapy in this group may reduce the reliability of the tuberculin skin test (TST) due to a high incidence of anergy. The value of interferon gamma release assays in this setting is also unclear. The aim of this study was to assess the value of a combination of the TST and Tspot for screening in patients on immunosuppressants and compare to the BTS-recommended approach, which advocates treatment decisions based on risk stratification.

Methods Adult patients referred for TB screening to a tertiary centre prior to commencement of Anti-TNF therapy were included in the study. All patients received a uniform screening protocol of clinical history, chest radiograph, stratification of TB risk (by ethnicity and age according to BTS guideline risk-tables), TST and Tspot.

Results There were 137 patients included with 116 (84.7\%) taking immunosuppressant medication at the time of screening. Of the sub-group on immunosuppressants, 17 patients (14.7\%) had positive TST results ( 5 patients with result $>15 \mathrm{~mm}$ in presence of BCG, 12 patients with result $>5 \mathrm{~mm}$ in absence of previous BCG). Of this sub-group with positive TST results, 12 patients (70.6\%) would not have been treated according to risk-stratification tables. Of the subgroup on immunosuppressants, 25 patients (21.6\%) had positive Tspot results and 1 patient had an indeterminate result.

Of this subgroup with positive Tspot results, 17 patients (68\%) would not have been treated according to risk-stratification tables.

When comparing strategies, use of risk-stratification tables alone would lead to 26 patients being treated with chemoprophylaxis. Addition of either positive TST and/or positive Tspot in patients not identified by risk stratification method led to an additional 22 patients eligible for treatment ( 9 patients Tspot positive alone; 7 patients TST positive alone; 6 patients double positive).

Conclusion Performing both a TST and Tspot in patients on immunosuppressants prior to commencement of Anti-TNF screening gives an additional yield of potential LTBI when risk assessment strategies alone would not have identified these.

\section{P57 COMPARATIVE COST-EFFECTIVENESS OF IGRA TO DETECT LATENT TB INFECTION IN UK INFLAMMATORY BOWEL DISEASE PATIENTS INITIATING ANTI-TNF $\alpha$ AGENTS}

doi:10.1136/thoraxjnl-2012-202678.198

${ }^{1} \mathrm{~K}$ Greveson, 'S Capocci, 'S Murthy, 'C Smith, 'S Morris, ${ }^{2} \mathrm{C}$ Murray, ${ }^{3} \mathrm{C}$ Cropley, ${ }^{2} \mathrm{M}$ Hamilton, ${ }^{1} \mathrm{M}$ Lipman. 'Department of Respiratory Medicine, Royal Free London NHS Foundation Trust, London, UK; ${ }^{2}$ Centre for Gastroenterology, Royal Free London NHS Foundation Trust, London, UK; ${ }^{3}$ Department of Infectious Diseases, Royal Free London NHS Foundation Trust, London, UK

Introduction Two commercial Interferon Gamma release assays (IGRA) are approved in the UK by NICE to detect $M$ tuberculosis (Mtb) infection. They use different test platforms. Both may provide borderline (neither clear positive or negative) or indeterminate (failure of control samples) results, especially in people taking immunomodulatory therapy. Since 2008, we have applied a standard IGRA-based assessment for latent Mtb infection in inflammatory bowel disease (IBD) patients being considered for anti-TNF $\alpha$ therapy. Initially this involved T-Spot.TB (TSTB) but in December 2010, our service switched to Quantiferon Gold In-tube (OFGIT). Here we review the performance and cost-effectiveness of these assays within our protocol.

Method Adult IBD patients were assessed using symptom review, chest radiograph (CXR) and IGRA Indeterminate/borderline IGRA were repeated and patients with persistently indeterminate/borderline results plus TB risk factors, or a positive result were referred to TB services. Cost per patient assessment used the average of assay costs and repeated assays plus onward referrals for those with indeterminate/borderline tests. Appointment costs were taken from 